\title{
Stability of phase locking in coupled semiconductor laser arrays
}

\author{
H. G. Winful and S. S. Wang \\ Deparment of Electrical Engineering and Computer Science, Universigy of Michigan, Ann Arbon, \\ Michigan 48109-2122
}

(Received 7 July 1988; accepted for publication 6 September 1988)

It is shown that amplitude phase coupling (as described by the linewidth enhancement factor

a) leads to unstable phase locking in semiconductor laser arrays with evanescent coupling.

The problem of synchronization of coupled oscillators is fundarnental to many areas of science. ${ }^{1}$ When synchronization is successful the several oscillators act as one, with a unique frequency and with well-defined phase relationships between the oscillators. When synchronization fails the behavior of the oscillators is marvelously complex. Here beat oscillations, quasi-periodic motions, and cheotic dynamics are the norm. Phase-locked semiconductor laser arrays are an important example of the complicated dynamics of coupled nonlinear oscillators. To date, however, there have been few experiments with the remporal resolution necessary to observe the interesting dynamics. ${ }^{2}{ }^{4}$ We recently presented numerical simulations which showed that the irregular and undamped spiking behavior observed by Elliots et al. ${ }^{2}$ is intrinsic to the laser arrays themselves. ${ }^{5}$ The temporal stability of semiconductor laser arrays appears questionable ${ }_{\varsigma}$ at best. In this letter we present a linear stability analysis that shows that laser arrays are unstable over large regions of a parameter space spanned by the coupling constant and the injection current. We point out the role of amplitude phase coupling, as described by the linewidth enhancement factor $\alpha$, in destabilizing plase locking between adjacent laser elements.

The dynamic behavior of semiconductor laser arrays with evanescent coupling is described by the equations":

$$
\begin{aligned}
\frac{d E_{i}}{d i}= & \frac{g}{2}\left(N_{i}-N_{\mathrm{th}}\right) E_{i}-\frac{\kappa c}{n}\left[E_{i+1} \sin \left(\phi_{i+1}-\phi_{i}\right)\right. \\
& \left.+E_{i-1} \sin \left(\phi_{i \ldots 1}-\phi_{i}\right)\right], \\
\frac{d \phi_{i}}{d t}= & -\frac{\alpha g^{\prime}}{2}\left(N_{i}-N_{\mathrm{th}}\right)+\frac{\kappa c}{n}\left[\frac{E_{i+1}}{E_{i}} \cos \left(\phi_{i+1}-\phi_{i}\right)\right. \\
& \left.+\frac{E_{i-1}}{E_{i}} \cos \left(\phi_{i \ldots 1}-\phi_{i}\right)\right],
\end{aligned}
$$

$\frac{d N_{i}}{d t}=P-\frac{N_{i}}{\tau_{s}}-\left(\frac{1}{\tau_{p}}+g^{\prime}\left(N_{i}-N_{\mathrm{th}}\right)\right) E_{i}^{2}$

where $N_{i}$ is the carrier density, $E_{i}$ is the amplitude, and $\phi_{j}$ is the phase of the electric field in the ith channel. The other parameters are the differential gain $g^{\prime}$ (suitabiy reduced by a mode confinement factor), the coupling constant $\kappa$, the threshold carrier density $N_{\text {th }}$, the linewidth enhancement factor $\alpha$, the pump rate $P$, the photon lifetime $\tau_{p}$, and the spontaneous carrier lifetime $\tau_{s}$. The phase-locked state, if it exists, is found by setting all the time derivatives to zero and solving for the steady-state values of $E_{i}, \phi_{i}$, and $N_{i}$. In what follows we show that for a wide range of parameters, the steady-state phase-locked state is unstable.

A stability analysis of Eqs. (1) - (3) is a daunting a ask for large $N$. For $N=2$, however, it is possible to obtain closedform expressions for the stability boundaries. For convenience we introduce the following rescaled variables and parameters:

$X_{i}=\left(\frac{1}{2} g^{\prime} \tau_{s}\right)^{1 / 2} E_{i}, \quad Z_{i}=(1 / 2) g^{\prime} N_{\mathrm{th}} \tau_{p}\left(N_{i} / N_{\mathrm{th}}-1\right)$

$p=(1 / 2) g^{i} N_{\mathrm{th}} \tau_{p}\left(P / P_{\mathrm{th}}-1\right), \quad \eta=(\kappa c / n) \tau_{p}$,

$T=\tau_{\mathrm{s}} / \tau_{p}$.

Then, for a two-element array, the coupled mode equations become

$$
\begin{aligned}
& \dot{X}_{1}=Z_{1} X_{1}-\eta X_{2} \sin \theta \\
& \dot{X}_{2}=Z_{2} X_{2}+\eta X_{1} \sin \theta \\
& \dot{\theta}=-\alpha\left(Z_{2}-Z_{1}\right)+\eta\left(X_{1} / X_{2}-X_{2} / X_{1}\right) \cos \theta, \\
& T \dot{Z}_{1}=p-Z_{1}-\left(1+2 Z_{1}\right) X_{1}^{2}, \\
& T \dot{Z}_{2}=p-Z_{2}-\left(1+2 Z_{2}\right) X_{2}^{2},
\end{aligned}
$$

where $\theta=\phi_{2}-\phi_{1}$ and the dots signify derivatives with respect to a reduced time $t / \tau_{p}$. Equations (4) possess the equilibria:

$$
\begin{aligned}
& \text { (I) } X_{1}=X_{2}=\sqrt{p}, \quad \mathbb{Z}_{1}=Z_{2}=0, \quad \theta=0, \\
& \text { (1I) } X_{1}=X_{2}=\sqrt{p}, \quad Z_{1}=Z_{2}=0, \quad \theta=\pi \text {. }
\end{aligned}
$$

To investigate the stability of these steady states we introduce snall perturbations and linearize Eqs. (4) about their steady-state values. The Routh-Hurwitz criterion is used to determine the regions of parameter space in which the steady-state solutions are stable. We find that the out-ofphase (II) solution is stable under the condition

$$
\eta<(1+2 p) / 2 \alpha T,
$$

while the in-phase (I) solution is stabie for

$$
\eta>a p /(1+2 p) \text {. }
$$

Figure 1 shows the instability domain in the plane of the variables $\eta$ (the coupling strength) and $p$ (the normalized excess pump current). The other key parameters are $\alpha=5$ and $T=2 \times 10^{3}$. It is clear that the phase-locked state is unstable over a wide region of the $\eta$ - $p$ plane. It should be noted that experimentai values of $\eta$ are in the range $10^{3}-$ $10^{-4}$ and that a value of $p=0.05$ corresponds to a pump current of order 1.3 times the threshold value. It is thus obvious that stability is assured only for very small values of $\eta$ in which case the lasers are essentially independent, or for 


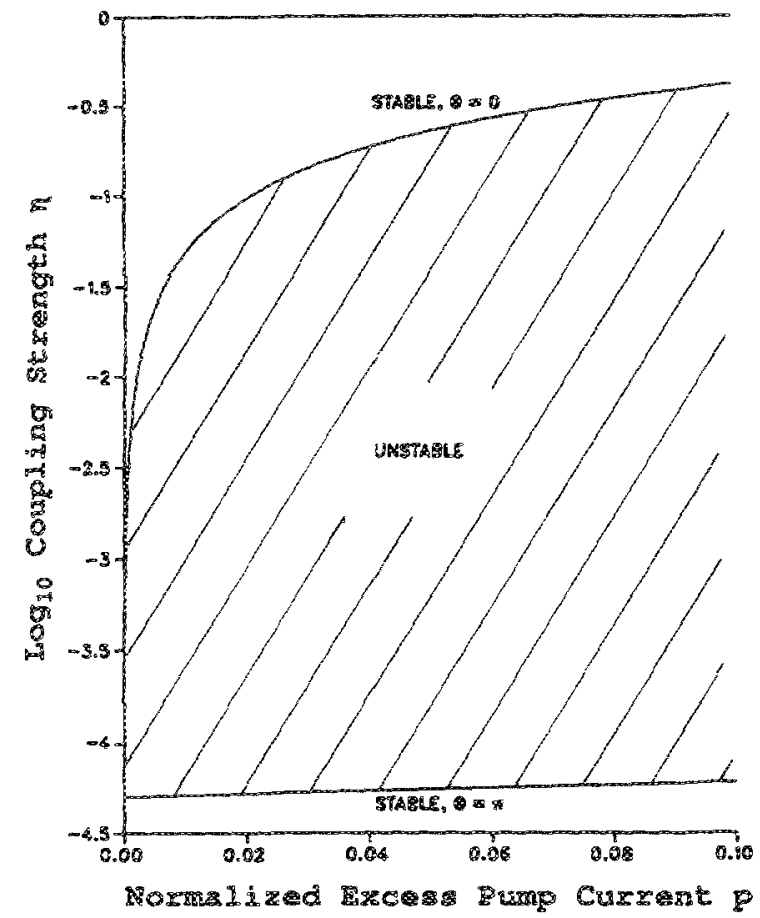

FIG. 1. Instability domain in the plane of the variables $\eta=\kappa \mathrm{c} \tau_{p} / n$, the coupling strength and $p=\left(\frac{1}{2} / 2\right) g^{\prime} N_{t h} \tau_{p}\left(P / P_{\text {th }}-1\right)$, the nornalized excess pump current.

very large values of $\eta$, in which case the two lasers are so tightly coupled that they act as one.

In the unstable regime the laser array exhibits sustained oscillations which may be periodic, quasi-periodic, or chaotic, depending on the values of the chosen parameters. Figure 2 shows time series of the amplitude and relative phase in the urstable region for $p=0.05$ and $\eta=10^{-4}$. The initial conditions were $X_{1}=X_{2}=\sqrt{p}, Z_{1}=Z_{2}=0$, and $\theta=0.1$. Note that the phases do not lock to a constant value. The phase difference oscillates about a mean value of $\theta=\pi$. Farfield measurements taken with averaging detectors will reveal a twin-lobed structure usually associated with out-ofphase locking. However, our analysis shows that the phases are not necessarily locked. It is important to note also that our analysis does not make any assumptions concerning the relative losses of the in-phase and out-of-phase modes. The preference of the systern to operate (on the average) in the out-of-phase mode is intrinsic to the nature of evanescent coupling and may be explained on the basis of a maximum emission principie. Near the stability boundaries, the linearized equations for the fuctuations yield an oscillation frequency given by ${ }^{7}$

$$
\omega=\left[\frac{1+g^{2} N_{0} \tau_{p}}{\tau_{p} \tau_{s}}\left(\frac{I}{I_{\mathrm{th}}}-1\right)+\left(\frac{\kappa c}{n}\right)^{2}\right]^{1 / 2}
$$

where $I$ is the injection current and $N_{t}=N_{t h}-1 / g^{\prime} \tau_{p}$. For small $\kappa$, the pulsation frequency has the characteristic dependence $\left(I / I_{\mathrm{th}}-1\right)^{1 / 2}$ associated with the relaxation os cillation resonance. However, when $\kappa$ is large, the pulsation frequency becomes simply the rate of energy transfer bet ween the lasers $\sim \kappa c / n$.

The origin of the instability is the amplitude phase cou-
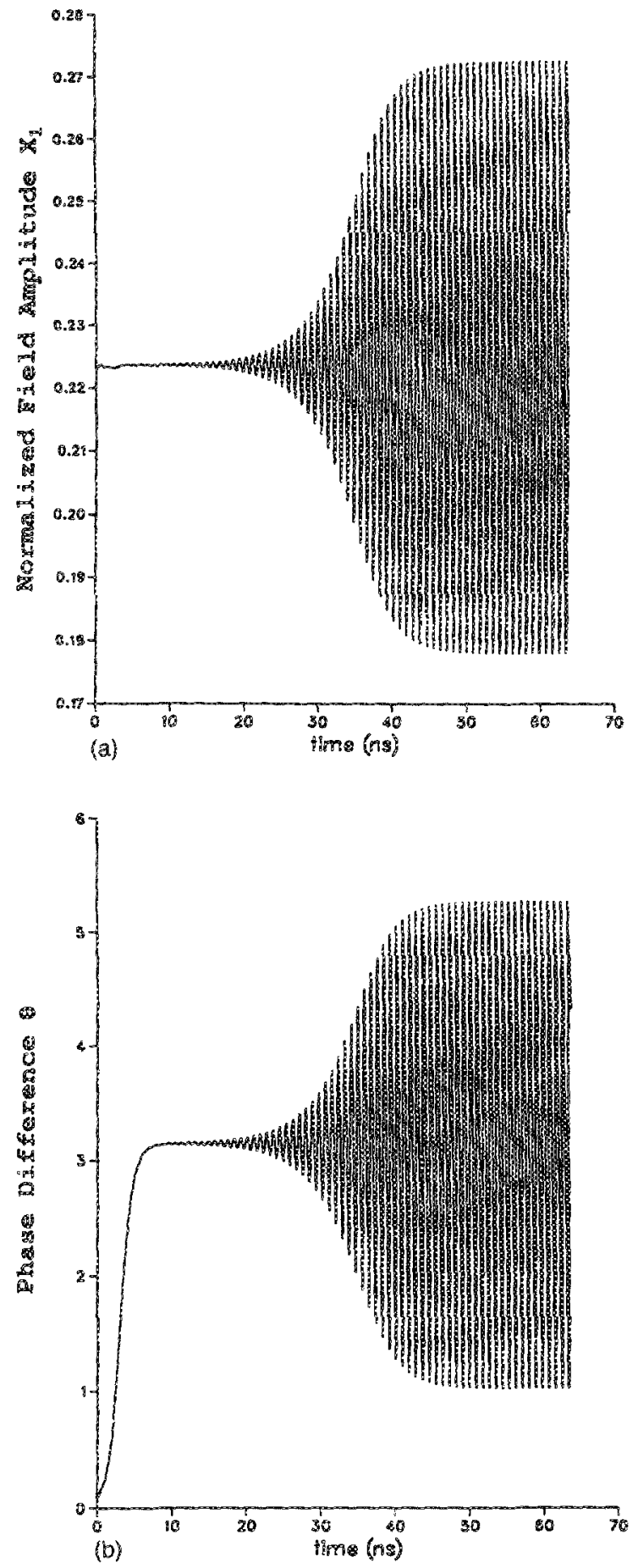

FG. 2. Periodic oscillations of (a) ficld amplitude and (b) relative phase in the unstable domain. Here $p=0.05$ and $\eta=10^{\circ}$. For larger values of $\eta$, the amplitude oscillations are aperiodic and the phase evolution is unbounded.

pling that occurs in semiconductor lasers and is described by the linewidth enhancement factor $\alpha$. Note that the instability disappears as $\alpha \rightarrow 0$. Also, the stability domain increases as $T \rightarrow 0$. An amplitude fiuctuation in one laser leads to a carrier density fuctuation and (through $\alpha$ ) a phase fluctuation in that same laser. A change in relative phase leads to an amplitude change in the second laser and an accompanying change in its carrier density. Sustained oscillations can be expected when the lifetime of the carriers $\left(\tau_{s}\right)$ is longer than or comparable to the coupling time $\left(\tau_{k}=n / \mathrm{kc}\right)$. 
The stability analysis presented here has shown that phase locking between adjacent laser elements car be destabilized by amplitude phase coupling. A similar result has been obtained in the problem of semiconductor laser injection locking. It is not yet clear how the presence of more than two lasers will affect the stability of the array. Numerical solutions of the array equations show that the instability persists for particular parameter vaiues. A general stability analysis for large $N$ is in progress. Also not included in the present analysis is the efiect of damping factors such as noninear gain and spontaneous emission into the iasing mode. These effects may help reduce the instability domain somewhat.

In conclusion, we have shown that amplitude phase coupling can lead to a self-pulsing instability in coherently coupled semiconductor lasers.
Partial support for this research was provided by the National Science Foundation through a Presidential Young Investigator Award (ECS-8657256) to H. Winful. Additional support through the Army Research Office is acknowledged.

'N. Minorsky, Nonlinear Oscillations (Van Nostrand, Princeton, NJ, 1962).

${ }^{2}$ R. A. Hliott, R. K. DeFreez, T. L. Paoli, R. D. Burnham, and W. Streifer, IEEE J. Quantum Electron. QE-21, 598 (1985).

${ }^{3}$ R. K. DeFreer, R. A. Ellott, K. Hartnet, and D. F. Weich, Electron. Lett. 23,589 (1987),

${ }^{4}$ K. A. Forest and J. B. Abshire, IEEE J. Quantum Electron. OE-23, 1287 (1987).

'S. S. Wang and H. G. Winful, Appl. Whys. Lett. 52, 1774 (1988).

${ }^{6} \mathrm{C}$. H. Henry, IEEE J. Quantum Electron. QE-18, 259 (1982).

7EI. G. Winful (unpublished).

${ }^{8}$ R. Lang, IFEE J. Quantum Electron. QE-18, 976 (1982), 